INPLASY

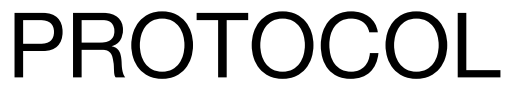

To cite: Yu et al. Efficacy and safety of acupuncture combined with Chinese Herbal Medicine for diabetic nephropathy: A protocol of systematic review and metaanalysis. Inplasy protocol 202180018. doi: 10.37766/inplasy2021.8.0018

Received: 05 August 2021

Published: 05 August 2021

Corresponding author: Zheng Nan

zy18543019097@163.com

Author Affiliation: Changchun University of Chinese Medicine

Support: 20190304054Y.

Review Stage at time of this submission: Preliminary searches.

Conflicts of interest: None declared.

\section{Efficacy and safety of acupuncture combined with Chinese Herbal Medicine for diabetic nephropathy: A protocol of systematic review and meta-analysis}

Yu, Z1; Zhang, W2; Li, B3; Bao, P4; Wang, F5; Sun, J6; Song, G7; Yin, L8; Nan, Z9.

Review question / Objective: This study aimed to conduct a meta-analysis of acupuncture combined with Chinese herbal medicine for the treatment of DN to clarify its efficacy. Condition being studied: Diabetic nephropathy (DN) is the most common cause of end-stage kidney disease. It is characterized by proteinuria, decline in glomerular filtration, hypertension, and a high risk of cardiovascular morbidity and mortality. As the epidemic of diabetes spreads, the number of patients at risk for developing $D N$ is increasing, which occurs in $20 \%$ to $40 \%$ of all diabetic patients. According to an epidemiological statistical report, the number of diabetic patients worldwide will reach 366 million in 2030, while the number of DN patients will exceed $\mathbf{1 0 0}$ million. Family and society have brought huge economic burden, but patients and their families have also brought greater psychological pressure.Acupuncture and Chinese herbal medicine treatments are often combined to treat DN; however, there has been no meta-analysis on their synergistic effect. Therefore, we aimed to perform a systematic review and meta-analysis to estimate the effectiveness of acupuncture combined with Chinese herbal medicine in DN treatment.

INPLASY registration number: This protocol was registered with the International Platform of Registered Systematic Review and Meta-Analysis Protocols (INPLASY) on 05 August 2021 and was last updated on 05 August 2021 (registration number INPLASY202180018).

\section{INTRODUCTION}

Review question / Objective: This study aimed to conduct a meta-analysis of acupuncture combined with Chinese herbal medicine for the treatment of DN to clarify its efficacy.

Condition being studied: Diabetic nephropathy (DN) is the most common cause of end-stage kidney disease. It is 
characterized by proteinuria, decline in glomerular filtration, hypertension, and a high risk of cardiovascular morbidity and mortality. As the epidemic of diabetes spreads, the number of patients at risk for developing DN is increasing, which occurs in $20 \%$ to $40 \%$ of all diabetic patients. According to an epidemiological statistical report, the number of diabetic patients worldwide will reach 366 million in 2030, while the number of DN patients will exceed $\mathbf{1 0 0}$ million. Family and society have brought huge economic burden, but patients and their families have also brought greater psychological pressure.Acupuncture and Chinese herbal medicine treatments are often combined to treat DN; however, there has been no metaanalysis on their synergistic effect. Therefore, we aimed to perform a systematic review and meta-analysis to estimate the effectiveness of acupuncture combined with Chinese herbal medicine in DN treatment.

\section{METHODS}

Participant or population: Participants who were definitively diagnosed with DN were included. No limitations of location, educational background, and gender were imposed.

Intervention: The intervention included acupuncture and Chinese herbal medicine. The control intervention included simple Western medicine, such as placebo or ACEI/ARB. Hypoglycemic therapy was used as a co-intervention in both arms, including oral hypoglycemic drugs, insulin, and exercise, or did not receive any treatment as a blank control.

Comparator: Intervention: placebo; no treatment; pharmacological compounds (for example such as irbesartan);nonpharmacological interventions (for example diet, exercise).

\section{Study designs to be included: RCTs.}

Eligibility criteria: Patients suffering from diabetic nephropathy.
Information sources: This study will use the Cochrane Library, Web of Science, PubMed, Embase, Allied and Complementary Medicine Database (AMED), China Biomedical Literature Database (CBM), China National Knowledge Infrastructure (CNKI), China Science and Technology Journal Database (VIP), Wanfang Database, and Ongoing Clinical Trials Database. There is no definite time limit for retrieval literature, and the languages will be limited to Chinese and English.

Main outcome(s): The primary outcomes included 24-hour urine protein quantitation, urinary albumin excretion rate, glomerular filtration rate, and fasting blood glucose level.

Quality assessment / Risk of bias analysis: Two investigators will separately assess the risk of bias of the selected RCTs using the Cochrane risk of bias assessment tool. The evaluation of each study mainly included the following seven aspects: random sequence generation, allocation hiding, blinding of participants and personnel, blinding of outcome assessment, incomplete outcome data, incomplete outcome data, selective outcome reporting, and other biases. Finally, the bias of the study will be rated on three levels: "low bias," "high bias," and "ambiguous bias. These even domains will be separately appraised by two reviews, and discrepancies will be addressed by consulting a third reviewer.

Strategy of data synthesis: In this study, we will apply RevMan 5.4 software for statistical analysis. The risk ratio and $95 \%$ confidence intervals (Cls) will be collected for enumeration data, while the mean difference or standardized mean difference and $95 \%$ Cls will be utilized to calculate continuous outcome data. The heterogeneity of the data was tested by calculating 12 statistics. The study was not considered to have a large heterogeneity when the 12 value was less than $50 \%$. when the 12 value exceeded $50 \%$, there was significant statistical heterogeneity among the trials. When there is homogeneity in the 
merged outcome results across sufficient studies, a meta-analysis will be conducted. Otherwise, we carried out a subgroup analysis to explore the causes of heterogeneity.

Subgroup analysis: We will investigate the source of heterogeneity using subgroup analysis based on different interventions, controls, and outcomes.

Sensitivity analysis: We will carry out a sensitivity analysis to investigate the robustness and stability of outcome results by removing low methodological quality studies. The main analysis points included the impact of method quality, sample size, and missing data on the study. In this way, we will be able to assess the impact of individual studies on the overall results and determine whether the results are strong.

Country(ies) involved: China.

Keywords: Chinese herbal medicine, traditional Chinese medicine, acupuncture, diabetic nephropathy, effectiveness, safety, protocol, systematic review.

Contributions of each author:

Author 1 - Ziyang Yu.

Email: zy18543019097@163.com

Author 2 - Wenfeng Zhang.

Email: 1139607280@qq.com

Author 3 - Borui Li.

Email: 513516742@qq.com

Author 4 - Pengjie Bao.

Email: baopengjie111@163.com

Author 5 - Fengyang Wang.

Email: wfy13159622688@126.com

Author 6 - Jian Sun.

Email: 173376400@qq.com

Author 7 - Guojiao Song.

Email: 815461810@qq.com

Author 8 - Lu Yin.

Author 9 - Zheng Nan. 